\title{
Validation that a 1-year fellowship in minimally invasive and bariatric surgery can eliminate the learning curve for laparoscopic gastric bypass
}

\author{
Mohamed R. Ali · David S. Tichansky · Shanu N. Kothari · Corrigan L. McBride \\ Adolfo Z. Fernandez Jr. • Harvey J. Sugerman · John M. Kellum • \\ Luke G. Wolfe · Eric J. DeMaria
}

Received: 11 April 2008/Accepted: 1 May 2009/Published online: 11 June 2009

(c) The Author(s) 2009. This article is published with open access at Springerlink.com

\begin{abstract}
Background The concept that advanced surgical training can reduce or eliminate the learning curve for complex procedures makes logical sense but is difficult to verify and has not been tested for laparoscopic Roux-en-Y gastric bypass (LRYGB). We sought to determine if minimally invasive/bariatric surgery fellowship graduates (FGs)
\end{abstract}

This study has been accepted for oral presentation at the annual meeting of the Society of American Gastrointestinal Endoscopic Surgeons (SAGES) in April 2008.

M. R. Ali ( ()

Department of Surgery, University of California, Davis, 2221 Stockton Boulevard, Sacramento, CA 95817, USA

e-mail: mohamed.ali@ucdmc.ucdavis.edu

D. S. Tichansky

Department of Surgery, Thomas Jefferson University,

Philadelphia, PA, USA

S. N. Kothari

Department of Surgery, Gundersen Lutheran Medical Center,

La Crosse, WI, USA

C. L. McBride

Department of Surgery, University of Nebraska Medical Center, Omaha, NE, USA

\author{
A. Z. Fernandez Jr. \\ Department of Surgery, Wake Forest School of Medicine, \\ Winston-Salem, NC, USA \\ H. J. Sugerman · J. M. Kellum · L. G. Wolfe \\ Department of Surgery, Virginia Commonwealth University, \\ Richmond, VA, USA \\ E. J. DeMaria \\ Department of Surgery, Duke University, Durham, NC, USA
}

would demonstrate complication-related outcomes (CRO) equivalent to the outcomes achieved during their training experience under the supervision of experienced bariatric surgeons.

Methods We compared CRO for the first 100 consecutive LRYGBs performed in practice by five consecutive minimally invasive/bariatric fellows at new institutions (total 500 cases) to CRO for the 611 consecutive LRYGBs performed during their fellowship training experience under the supervision of three experienced bariatric surgeons at the host training institution.

Results The two patient groups did not differ demographically. The 18 types of major and minor complications identified after LRYGB did not differ among the five fellowship graduates. The mentors' CRO were compatible with published benchmark data. As compared with the training institution data, the overall incidence of complications for the combined experience of fellowship graduates did not differ statistically from that of the mentors. The fellowship graduates' early experience included zero nongastrojejunostomy leaks ( $0 \%$ versus $1.5 \%)$ and a low rate of anastomotic stricture $(0.8 \%$ versus $3.0 \%)$, incisional hernia ( $1 \%$ versus $4.4 \%)$, bowel obstruction $(0 \%$ versus $3 \%$ ), wound infection $(0.3 \%$ versus $3.1 \%)$, and gastrointestinal hemorrhage $(0.2 \%$ versus $1.6 \%)$. The rate of gastrojejunostomy leak (1.8\% versus $2.6 \%)$ and, most importantly, mortality $(0.8 \%$ versus $0.7 \%)$ did not differ between the two groups.

Conclusions Fellowship graduates achieved high-quality surgical outcomes from the very beginning of their postfellowship practices, which are comparable to those of their experienced mentors. These data validate the concept that advanced surgical training can eliminate the learning curve often associated with complex minimally invasive procedures, specifically LRYGB. 
Keywords Bariatric surgery - Laparoscopy - Obesity . Gastric bypass - Fellowship training .

Minimally invasive surgery

Class III obesity and its associated medical conditions continue to pose serious public health concerns in the USA [1]. The extraordinary efficacy of gastric bypass to achieve and maintain significant weight loss has been well established [2-4]. Over the past decade, the increasing application of minimally invasive techniques has revolutionized bariatric surgery. Within this time frame, the annual number of laparoscopic Roux-en-Y gastric bypass (LRYGB) operations performed in the USA has risen dramatically.

LRYGB is a technically challenging operation that can be associated with serious intraoperative and postoperative complications $[5,6]$. Reduction in morbidity and mortality associated with LRYGB has been shown to occur as a function of surgical experience [7-9]. This important issue has been recognized by the American Society for Metabolic and Bariatric Surgery (ASMBS) in its published Guidelines for Granting Privileges in Bariatric Surgery [10]. The ASMBS recommends that surgeons should demonstrate an experience of 50 LRYGB cases with successful outcomes to be privileged to perform this operation.

There are a number of avenues by which surgeons can gain instruction regarding LRYGB [11-13]. Laparoscopic bariatric workshops have proven an important source of introductory education [11]. However, these courses cannot provide the operative skill development and experience required to overcome the steep learning curve for LRYGB [11]. Mini-fellowships, offered over a period of weeks, allow the trainee to implement a bariatric surgery program but also may not meet every trainee's needs and likely do not eliminate the learning curve [12].

Indeed, the learning curve for LRYGB is steep [14, 15]. As a result, fellowship training in minimally invasive and bariatric surgery has increased in popularity and availability. While it may seem intuitive that devotion of time to a 1-year fellowship makes logical sense, some remaining issues must be resolved in order to establish the efficacy of this training paradigm for minimally invasive and bariatric surgery. First, given the technical complexity of LRYGB, does initiation of a fellowship program negatively impact the surgical outcomes at the mentoring institution (MI)? At least one previous study suggests that this negative impact does not occur [16]. A more complex issue for study is whether or not fellowship training can eliminate the learning curve for LRYGB when the fellowship graduate (FG) relocates to a new institution. Intertwined within these issues is the intricacy of comprehensive bariatric decision-making in selecting patients, managing complications, providing long-term aftercare, etc. In fact, FGs, who matriculate to a facility without a previously established bariatric surgery program face numerous logistical issues, including recruitment and training of support staff in the operating room and across the spectrum of outpatient and inpatient care. Furthermore, graduates who develop their own programs may be, in essence, "working without a net" in that they have to provide all necessary expertise in the absence of an on-site mentor, experienced in minimally invasive bariatric surgery, to provide counsel, experience, support, back-up, etc. To date, isolated small series have suggested that fellowship-trained minimally invasive bariatric surgeons perform well in practice [13, 17, 18].

We sought to determine whether graduates of a fellowship program in minimally invasive and bariatric surgery would demonstrate complication-related outcomes (CRO) equivalent to the outcomes achieved during their training experience under the supervision of experienced bariatric surgeons.

\section{Materials and methods}

\section{Study population}

Between July 2000 and June 2003, five surgeons (M.R.A, C.L.M., S.N.K., D.S.T., and A.Z.F.) trained as fellows in minimally invasive and bariatric surgery at Virginia Commonwealth University under the supervision of three experienced bariatric surgeons (H.J.S., E.J.D., and J.M.K.). All consecutive LRYGBs, performed during this period of training, were compared with the first 100 LRYGBs performed by each FG, after completion of training, in terms of complication-related outcomes (CRO). CRO data were obtained for all patients in both groups for the first 12 months following LRYGB. Postoperative follow-up was accomplished per the protocol of each FG and the MI and included clinic visits, telephone encounters, support groups, and correspondence. One year of follow-up data was obtained for all patients in this study. All patients included in this study met the criteria for bariatric surgery as a medical necessity as established by the National Institutes of Health [19]. There were no exclusion criteria.

\section{Surgical technique}

All patients were evaluated by the respective surgeon to determine the patient's candidacy for LRYGB. Preoperative workup was performed according to the protocol followed by each surgeon but commonly included new patient orientation, physiologic screening, psychological evaluation, and nutritional counseling. 
All patients included in this study were scheduled to undergo LRYGB. The operative technique consisted of a completely stapled jejunojejunostomy (JJ) for all surgeons, with varying lengths and paths (retrocolic or antecolic) of the alimentary limb (Table 1). All surgeons created a stapled gastric pouch of $20-30 \mathrm{~mL}$ in volume with a linearstapled gastrojejunostomy (GJ) and intraoperatively tested this anastomosis for leakage (Table 1).

\section{Data management}

Each FG entered data regarding patient demographics, surgical technique, relevant patient management protocols, and complications into a database using a standardized electronic form. Similar data were extracted from a prospective database kept at the MI. These entries were aggregated, without any patient identifiers, for statistical analysis to compare the five individual FG experiences as well as the aggregate experiences of the FGs and the MI. Each investigator managed data contribution according to local Institutional Review Board policies and procedures. Student's $t$-test was used to analyze pairs of continuous data sets. Multiple comparisons of continuous variables were accomplished with analysis of variance (ANOVA), using the Bonferroni post-test to evaluate significance between specific pairs of means. Discrete variables were tested using Fisher's exact test. Statistical significance was set at $\alpha=0.05$ for all analyses. Power analysis at this $\alpha$ level indicated that this study was adequately powered to detect a difference of $5 \%$ with a power of 0.8 and a difference of $5.5 \%$ with a power of 0.9 . We felt that the ability to identify these small differences was biologically valid.

\section{Results}

The early experience of each FG was similar (Table 1). The gender distribution of the five groups of patients was statistically similar and demonstrated a preponderance of female patients as is often seen in a population of bariatric surgery patients (Table 1). Although not available for surgeon 5, the mean American Society of Anesthesiologists (ASA) classification did not differ significantly among patients of the other four surgeons. The mean age of patients operated by surgeon 4 was higher than the other groups $(p<0.05)$, while the mean body mass index (BMI) of patients operated by surgeon 1 was lower than that of surgeons 2 and $4(p<0.05)$ (Table 1$)$.

During training, FGs had participated as primary surgeon in an average of $104 \mathrm{LRYGB}$ procedures with a range of case experience between 72 and 127 procedures. Over the training period for the FGs, 611 LRYGB operations were preformed at the MI. Of these cases, $520(85 \%)$ were performed with one of the FGs as primary surgeon (Table 1). The remaining

Table 1 Patient characteristics and surgical technique

Surgeon $1(n=100) \quad$ Surgeon $2(n=100) \quad$ Surgeon $3(n=100) \quad$ Surgeon $4(n=100) \quad$ Surgeon $5(n=100)$

\section{Patient factors}

Age (range), years

BMI $\left(\mathrm{kg} / \mathrm{m}^{2}\right)$ (range)

ASA (range)

Female (\%)

Male (\%)

Technical factors

Laparoscopic (\%)

Converted to open $(\%)$

$\mathrm{JJ}$ technique

Primary Roux limb path

Primary GJ technique

Intraop. leak test

Intraop. endoscopy

DVT prophylaxis

Port-site closure

No. of cases as fellow

$\begin{array}{ll}42(21-59)^{*} & 44(25-69)^{*} \\ 46.6(35-64)^{* *} & 50.7(38-72) \\ 2.6(2-3) & 2.8(2-4) \\ 94(94 \%) & 85(85 \%) \\ 6(6 \%) & 15(15 \%)\end{array}$

$99(99 \%)$

$1(1 \%)$

stapled

retro/retro

Two-layer linear

Air

Yes

SCD + LMWH

$10 \mathrm{~mm}$ and larger

98

$96(96 \%)$

$4(4 \%)$

stapled

retro/retro

Air

Yes

72
Two-layer linear

SCD + LMWH

$10 \mathrm{~mm}$ and larger

$41(23-64)^{*}$
$48.6(37-64)$
$2.2(1-3)$
$87(87 \%)$
$13(13 \%)$

\section{9 (21-70) \\ 50.7 (36-93) \\ $2.4(2-3)$ \\ $85(85 \%)$ \\ $15(15 \%)$}

$100(100 \%)$

$0(0 \%)$

stapled

retro/retro

Two-layer linear

Air

Yes

$\mathrm{SCD}+\mathrm{LMWH}$

None

102

$100(100 \%)$
$0(0 \%)$
stapled

ante/ante

Two-layer linear

Methylene Blue

No

$\mathrm{SCD}+\mathrm{LMWH}$

None

127

$42(24-66)^{*}$
$50.0(36-72)$
N/A
$89(89 \%)$
$11(11 \%)$
$97(97 \%)$
$3(3 \%)$
stapled
ante/ante
Two-layer linear
Air
Yes
SCD + LMWH
None
121

$42(24-66) *$

N/A

$89(89 \%)$

$11(11 \%)$

$97(97 \%)$

$3(3 \%)$

stapled

Two-layer linear

Air

121

$B M I$ body mass index, $A S A$ American society of anesthesiologists classification, $J J$ jejunojejunostomy, $G J$ gastrojejunostomy, $D V T$ deep vein thrombosis, $S C D$ sequential compression device, $L M W H$ low-molecular-weight heparin

* Significant difference compared with surgeon $4(p<0.05)$

** Significant difference compared with surgeons 2 and $4(p<0.05)$ 
91 cases were performed either with an FG as assistant or without a fellow at all. One of the attending surgeons was present during all procedures at the MI. In postgraduate practice, FGs were successful in completing gastric bypass procedures via laparoscopic technique, without open conversion, between $96 \%$ and $100 \%$ of the time (Table 1). The overall open conversion rate for the FGs did not differ from that of the MI (1.6\% versus $1.3 \%$, Table 2$)$.

Not surprisingly, review of the initial programmatic experience of each fellow revealed that FGs had adopted many of the algorithms for patient care and techniques for surgery utilized at the MI. All FGs used pharmacologic as well as mechanical prophylaxis against deep vein thrombosis (DVT). All FGs fashioned a stapled JJ, using techniques similar to those at the MI. During their training at the MI, the FGs were exposed to various techniques for creation of the GJ (circular stapling, linear stapling, and handsewn). Ultimately, the linear-stapled technique evolved to be the most popular at the MI. FGs also adopted this technique and uniformly reported creation of the GJ using a two-layer linear-stapled technique and testing the
GJ intraoperatively for leakage, as routinely done at the MI (Table 1). Similar to at the MI, four FGs routinely performed intraoperative upper endoscopy to accomplish this test for leakage (surgeon 4 did not), and only surgeons 1 and 2 routinely closed all laparoscopic port sites (Table 1). During training, FGs gained experience in both retrocolic retrogastric passage of the alimentary limb as well as the antecolic antegastric technique with omental division. In practice, the route of the alimentary limb similarly varied according to the preference of the individual FG (Table 1).

Demographically, the combined group of all FG patients $(n=500)$ was similar to the MI patients $(n=611)$ in terms of mean BMI and in gender distribution (Table 2). However, the mean age of FG patients was significantly older than the MI patients (43.9 versus 42.0 years, $p<0.05$ ), but this small difference is of questionable clinical significance. The prevalence of specific complications and overall mortality for LRYGB did not differ significantly between the reported experiences of the five FGs during their first 100 cases after training. A total of 18 major and minor complications were tracked and reported

Table 2 Comparison of demographic factors and complications

\begin{tabular}{|c|c|c|c|}
\hline & Fellows $(n=500)$ & Mentors $(n=611)$ & Significance \\
\hline \multicolumn{4}{|l|}{ Demographics } \\
\hline Age (range), years & $43.9(21-70)$ & $42.0(15-67)$ & $p<0.05$ \\
\hline BMI $\left(\mathrm{kg} / \mathrm{m}^{2}\right)$ (range) & $49.3(35-93)$ & $48.9(36-86)$ & NS \\
\hline Female $(\%)$ & $440(88 \%)$ & $524(86 \%)$ & NS \\
\hline Converted to open $(\%)$ & $8(1.6 \%)$ & $8(1.3 \%)$ & NS \\
\hline \multicolumn{4}{|l|}{ Complications } \\
\hline Marginal ulcer & $28(5.6 \%)$ & $33(5.4 \%)$ & NS \\
\hline GJ leak & $9(1.8 \%)$ & $16(2.6 \%)$ & NS \\
\hline GG fistula & $2(0.4 \%)$ & $1(0.2 \%)$ & NS \\
\hline GJ stricture & $4(0.8 \%)$ & $18(2.9 \%)$ & $p<0.05$ \\
\hline Non-GJ leak & 0 & $9(1.5 \%)$ & $p<0.05$ \\
\hline $\mathrm{JJ}$ obstruction & $4(0.8 \%)$ & $1(0.2 \%)$ & NS \\
\hline Internal hernia & $7(1.4 \%)$ & $12(2.0 \%)$ & NS \\
\hline $\mathrm{PE}$ & $3(0.6 \%)$ & $4(0.7 \%)$ & NS \\
\hline Bowel obstruction & 0 & $18(2.9 \%)$ & $p<0.05$ \\
\hline Incisional hernia & $5(1.0 \%)$ & $26(4.3 \%)$ & $p<0.05$ \\
\hline Wound infection & $15(3.0 \%)$ & $2(0.3 \%)$ & $p<0.05$ \\
\hline GI hemorrhage & $8(1.6 \%)$ & $1(0.2 \%)$ & $p<0.05$ \\
\hline Other* & $8(1.6 \%)$ & N/A & N/A \\
\hline Total complications & $93(18.6 \%)$ & $141(23.1 \%)$ & NS \\
\hline Mortality & $4(0.8 \%)$ & $4(0.7 \%)$ & NS \\
\hline
\end{tabular}

* Complications reported by the fellow graduates but not tracked in the data of the mentor institution. These complications are emergency reintubation (1), acute respiratory distress syndrome (1), multiple organ system failure (2), brachial plexus injury (1), splenic infarction (1), and deep vein thrombosis (2)

$B M I$ body mass index, $G J$ gastrojejunostomy, $G G$ gastrogastric, $J J$ jejunojejunostomy, $P E$ pulmonary embolism, GI gastrointestinal, $A R D S$ acute respiratory distress syndrome, $M O S F$ multiple organ system failure, $D V T$ deep vein thrombosis, $N S$ not significant 
by the FGs (Table 2). Six of these 18 complications, uncommon in the FG group, were not prospectively tracked as discrete variables by the MI. These six complications were therefore not available for comparison between the FGs and MI and included emergency reintubation, acute respiratory distress syndrome (ARDS), multiple organ system failure, brachial plexus injury, splenic infarction, and lower-extremity deep vein thrombosis (Table 2).

The remaining 12 complications were compared between FGs and MI. Most major complications and overall mortality $(0.7 \%$ versus $0.8 \%)$ did not differ between the early experience reported by the five FGs and the data collected prospectively by the MI (Table 2). The total complication rate, including both perioperative and delayed complications, did not differ between the two groups (18.6\% versus $23.1 \%$ ). Major life-threatening perioperative complications, including pulmonary embolism $(0.6 \%$ versus $0.7 \%)$ and GJ leak (1.8\% versus $2.6 \%$ ), did not differ between FGs and the MI. In contrast, there was a higher incidence of non-GJ leak reported by the MI $(1.5 \%$ versus $0 \%, p<0.05)$, while the MI identified a lower rate of postoperative gastrointestinal bleeding than that reported by the FGs $(0.2 \%$ versus $1.6 \%$, $p<0.05)$. Postoperative obstruction at the JJ did not differ between the two groups $(0.8 \%$ versus $0.2 \%)$. Postoperative wound infection was more common in the FG experience (3.0\% versus $0.3 \%, p<0.05)$

In terms of delayed postoperative complications, there was no statistically significant difference in marginal ulcer formation $(5.6 \%$ versus $5.4 \%)$, internal hernia (1.4\% versus $2.0 \%$ ) or in the development of gastrogastric (GG) fistula $(0.4 \%$ versus $0.2 \%$ ) between the FGs and the MI. The FGs did report a lower incidence of GJ stricture $(0.8 \%$ versus $2.9 \%$, $p<0.05$ ), fewer bowel obstructions (0\% versus $2.9 \%$, $p<0.05)$, and fewer incisional hernias $(1.0 \%$ versus $4.3 \%$, $p<0.05)$.

Upon completion of fellowship training, three FGs initiated a new program for laparoscopic performance of gastric bypass while two FGs joined established programs staffed by surgeons with at least some experience in performing LRYGB. These two subgroups of FGs demonstrated similar patient selection, although FGs that joined an established program operated on patients with higher mean BMI than those starting new programs (Table 3 ). $\mathrm{CRO}$, however, were no different between the two subgroups of FGs. Similar to the above comparisons, complications experienced by the subgroup of FGs initiating a new program did not differ from those tracked by the MI (Table 3).

\section{Discussion}

The history of surgery is marked by constant pursuit of excellence in quality of patient care. Within this context, substantial consideration has been given to the methods by which surgical education is delivered to trainees [20, 21]. With the expansion of the surgical sciences, specialized post-residency training has become commonplace.

Coincident with this increasing specialization of surgeons has come a tremendous upsurge in the popularity of bariatric surgery. This trend has been fueled by the safe and efficacious application of minimally invasive techniques to time-honored operations and the effectiveness and durability of surgery for weight-loss maintenance and comorbidity resolution [22-24]. While clinically effective, bariatric surgery poses significant clinical and technical challenges to surgeons. Bariatric surgery mixes the technical demand of complex operations with the clinical challenges of adequately preparing the bariatric patient for safe surgery and appropriately managing the patient postoperatively. Specifically, LRYGB has repeatedly been associated with a significant learning curve $[14,15,17,18]$. Moreover, surgical outcomes improve when the learning curve has been surpassed [14, 15, 17, 18].

It has been suggested that fellowship training in minimally invasive and bariatric surgery can attenuate the learning curve for RYGB. However, this phenomenon has not been conclusively determined, with two issues remaining at the heart of this debate: (1) Can surgical mentors safely absorb the learning curve of trainees? (2) Can fellowship training eliminate the post-training learning curve for trainees?

In this study, graduates of a fellowship training program in minimally invasive and bariatric surgery were able to achieve excellent operative outcomes with LRYGB. Regarding experience with LRYGB at the MI, one faculty mentor (E.J.D.) had begun performance of LRYGB in 1998, 2 years before initiation of the fellowship program. The other two mentor faculty, both with extensive experience with open gastric bypass over many years, began to perform LRYGB closer to initiation of the fellowship, with the assistance of their experienced colleague. While it is possible that the MI faculty had not completely climbed their own learning curves for LRYGB during the early period of fellowship training and that this could represent a bias in the current study, the MI demonstrated CRO for LRYGB comparable to other published benchmark data for cases performed during the period of training [25]. Specifically, the rates of significant complications for the MI, detailed in Table 2, were within the ranges established in landmark publications describing experience with LRYGB [26-28]. The MI rate of GJ leak (2.6\%) falls within the range of $0-3.6 \%$ established in these studies [26-28]. Internal hernia occurred in $2.0 \%$ of MI patients and in $0.3-$ $3.0 \%$ of cases in these studies [27, 28]. Non-GJ leak occurred in $1.5 \%$ (reference range from benchmark data: $1.5-1.8 \%$ ) of patients at the MI, and pulmonary embolism 
Table 3 Outcome data by type of practice joined by FG

\begin{tabular}{|c|c|c|c|}
\hline & New $(n=300)$ & Established $(n=200)$ & Mentors $(n=611)$ \\
\hline \multicolumn{4}{|l|}{ Demographics } \\
\hline Age (range), years & $44.4(21-70)^{1}$ & $43.2(24-69)$ & $42.0(15-67)$ \\
\hline BMI $\left(\mathrm{kg} / \mathrm{m}^{2}\right)$ (range) & $48.4(35-72)$ & $50.7(36-93)^{2}$ & $48.9(36-86)$ \\
\hline Female $(\%)$ & $270(90 \%)$ & $170(85 \%)$ & $524(86 \%)$ \\
\hline Converted to open $(\%)$ & $5(1.7 \%)$ & $3(1.5 \%)$ & $8(1.3 \%)$ \\
\hline \multicolumn{4}{|l|}{ Complications } \\
\hline Marginal ulcer & $16(5.3 \%)$ & $12(6.0 \%)$ & $33(5.4 \%)$ \\
\hline GJ leak & $5(1.7 \%)$ & $4(2.0 \%)$ & $16(2.6 \%)$ \\
\hline GG fistula & $1(0.3 \%)$ & $1(0.5 \%)$ & $1(0.2 \%)$ \\
\hline GJ stricture & $1(0.3 \%)^{1}$ & $3(1.5 \%)$ & $18(2.9 \%)$ \\
\hline Non-GJ leak & $0^{1}$ & 0 & $9(1.5 \%)$ \\
\hline JJ obstruction & $4(1.3 \%)^{1}$ & 0 & $1(0.2 \%)$ \\
\hline Internal hernia & $6(2.0 \%)$ & $1(0.5 \%)$ & $12(2.0 \%)$ \\
\hline $\mathrm{PE}$ & 0 & $3(1.5 \%)$ & $4(0.7 \%)$ \\
\hline Bowel obstruction & $0^{1}$ & $0^{1}$ & $18(2.9 \%)$ \\
\hline Incisional hernia & $2(0.7 \%)^{1}$ & $3(1.5 \%)$ & $26(4.3 \%)$ \\
\hline Wound infection & $8(2.7 \%)^{1}$ & $7(3.5 \%)^{1}$ & $2(0.3 \%)$ \\
\hline GI hemorrhage & $6(2.0 \%)^{1}$ & $2(1.0 \%)$ & $1(0.2 \%)$ \\
\hline Other* & $6(2.0 \%)$ & $2(1.0 \%)$ & N/A \\
\hline Total complications & $55(18.3 \%)$ & $38(19.0 \%)$ & $141(23.1 \%)$ \\
\hline Mortality & $1(0.3 \%)$ & $3(1.5 \%)$ & $4(0.7 \%)$ \\
\hline
\end{tabular}

1 Significant difference compared with mentors $(p<0.05)$

2 Significant difference compared with new programs and mentors $(p<0.05)$

* Complications reported by the fellow graduates but not tracked in the data of the mentor institution. These complications are emergency reintubation (1), acute respiratory distress syndrome (1), multiple organ system failure (2), brachial plexus injury (1), splenic infarction (1), and deep vein thrombosis (2)

$B M I$ body mass index, $G J$ gastrojejunostomy, $G G$ gastrogastric, $J J$ jejunojejunostomy, $P E$ pulmonary embolism, $G I$ gastrointestinal, $A R D S$ acute respiratory distress syndrome, MOSF multiple organ system failure, $D V T$ deep vein thrombosis

occurred in $0.7 \%$ of patients (reference range from benchmark data: $0-0.7 \%$ ) [26-28]. Similarly, $0.2 \%$ of MI patients developed JJ obstruction and $0.2 \%$ developed GI hemorrhage as compared with published rates of $0.7 \%$ for $\mathrm{JJ}$ obstruction and $0.3-0.8 \%$ for GI hemorrhage [26-28]. We interpret this as supportive evidence, as demonstrated by other investigators [16], that fellowship training does not adversely impact the CRO of the mentor program and that the MI safely absorbed the learning curves of the FGs without compromising patient care in this study.

In this study, we performed an exhaustive search for complications and mortality, within the first postoperative year, for the first 100 cases performed by five FGs in their immediate postgraduation new practice environments. All FGs demonstrated high-quality surgical outcomes for LRYGB, similar to those of the experienced surgeons at the MI, signifying that the steep learning curve for LRYGB had been eliminated. Most impressive was the observation that three of the FGs initiated a new program in laparoscopic gastric bypass immediately after completion of training and that this subgroup, working without available local expertise in LRYGB, was able to achieve excellent technical results and CRO comparable to benchmark data.

High-quality surgical outcomes, from the beginning of clinical practice, were achieved by FGs, despite differences in LRYGB experience during fellowship (ranging between 72 and 127 cases). Of note, all trainees exceeded the 50 LRYGB case experience threshold for hospital credentialing, as recommended by the ASMBS, during their fellowship. Since all FGs, in the current study, exceeded this case volume standard proposed by the ASMBS, it is possible to interpret these results as evidence that a higher level of case experience should be required to achieve benchmark outcomes, as reported herein. Although this study was not designed to determine what number of cases is sufficient experience for training in LRYGB, it is clear that experience with an average of 100 cases over the course of 1 year of advanced fellowship training (including comprehensive patient management education as well as skill training) was sufficient to allow FGs to achieve 
quality outcomes in practice. It is possible that a lower number of cases would have also provided sufficient experience, particularly with a comprehensive curriculum in place or for trainees who already possess advanced laparoscopic skills.

Graduates of surgery training programs can expect to face a new learning associated with independent post-training operative practice, which can be particularly steep for complex minimally invasive procedures such as LRYGB. Our data show that 1 year of minimally invasive and bariatric surgery fellowship education can effectively and reproducibly prepare trainees to achieve excellent CRO for LRYGB, often considered one of the most technically challenging minimally invasive procedures, from the very beginning of their post-training experience. Thus, this study validates the concept that advanced post-residency training can eliminate the post-training learning curve associated with complex minimally invasive procedures, in general. Furthermore, such training in the complex specialty of bariatric surgery can specifically eliminate the steep learning curve for laparoscopic gastric bypass surgery, as frequently encountered in independent surgical practice.

Open Access This article is distributed under the terms of the Creative Commons Attribution Noncommercial License which permits any noncommercial use, distribution, and reproduction in any medium, provided the original author(s) and source are credited.

\section{References}

1. Wang Y, Beydoun MA (2007) The obesity epidemic in the United States-gender, age, socioeconomic, racial/ethnic, and geographic characteristics: a systematic review and metaregression analysis. Epidemiol Rev 29:6-28

2. Peluso L, Vanek VW (2007) Efficacy of gastric bypass in the treatment of obesity-related comorbidities. Nutr Clin Pract 22:22-28

3. Velcu LM, Adolphine R, Mourelo R, Cottam DR, Angus LD (2005) Weight loss, quality of life and employment status after Roux-en-Y gastric bypass: 5-year analysis. Surg Obes Relat Dis 1:413-416 discussion 417

4. Ali MR, Fuller WD, Choi MP, Wolfe BM (2005) Bariatric surgical outcomes. Surg Clin North Am 85:835-852 vii

5. Gould JC, Garren MJ, Starling JR (2004) Lessons learned from the first 100 cases in a new minimally invasive bariatric surgery program. Obes Surg 14:618-625

6. Nguyen NT, Huerta S, Gelfand D, Stevens CM, Jim J (2004) Bowel obstruction after laparoscopic Roux-en-Y gastric bypass. Obes Surg 14:190-196

7. Hsu GP, Morton JM, Jin L, Safadi BY, Satterwhite TS, Curet MJ (2005) Laparoscopic Roux-en-Y gastric bypass: differences in outcome between attendings and assistants of different training backgrounds. Obes Surg 15:1104-1110

8. Lublin M, Lyass S, Lahmann B, Cunneen SA, Khalili TM, Elashoff JD et al (2005) Leveling the learning curve for laparoscopic bariatric surgery. Surg Endosc 19:845-848

9. Shikora SA, Kim JJ, Tarnoff ME, Raskin E, Shore R (2005) Laparoscopic Roux-en-Y gastric bypass: results and learning curve of a high-volume academic program. Arch Surg 140:362-367
10. Chau WY, Schmidt HJ, Kouli W, Davis D, Wasielewski A, Ballantyne GH (2005) Patient characteristics impacting excess weight loss following laparoscopic adjustable gastric banding. Obes Surg 15:346-350

11. Lord JL, Cottam DR, Dallal RM, Mattar SG, Watson AR, Glasscock JM et al (2006) The impact of laparoscopic bariatric workshops on the practice patterns of surgeons. Surg Endosc 20:929-933

12. Cottam D, Holover S, Mattar SG, Sharma SK, Medlin W, Ramanathan R et al (2007) The mini-fellowship concept: a six-week focused training program for minimally invasive bariatric surgery. Surg Endosc 21:2237-2239

13. McIntyre T, Jones DB (2005) Training methods for minimally invasive bariatric surgery. Surg Technol Int 14:57-60

14. Oliak D, Ballantyne GH, Weber P, Wasielewski A, Davies RJ, Schmidt HJ (2003) Laparoscopic Roux-en-Y gastric bypass: defining the learning curve. Surg Endosc 17:405-408

15. Schauer P, Ikramuddin S, Hamad G, Gourash W (2003) The learning curve for laparoscopic Roux-en-Y gastric bypass is 100 cases. Surg Endosc 17:212-215

16. Gonzalez R, Nelson LG, Murr MM (2007) Does establishing a bariatric surgery fellowship training program influence operative outcomes? Surg Endosc 21:109-114

17. Kothari SN, Boyd WC, Larson CA, Gustafson HL, Lambert PJ, Mathiason MA (2005) Training of a minimally invasive bariatric surgeon: are laparoscopic fellowships the answer? Obes Surg 15:323-329

18. Oliak D, Owens M, Schmidt HJ (2004) Impact of fellowship training on the learning curve for laparoscopic gastric bypass. Obes Surg 14:197-200

19. Anonymous (1992) Gastrointestinal surgery for severe obesity: national institutes of health consensus development conference statement. Am J Clin Nutr 55:615S-619S

20. Moulton CA, Dubrowski A, Macrae H, Graham B, Grober E, Reznick R (2006) Teaching surgical skills: what kind of practice makes perfect?: a randomized, controlled trial. Ann Surg 244:400-409

21. Grober ED, Jewett MA (2006) The concept and trajectory of "operative competence" in surgical training. Can J Surg 49:238-240

22. McNatt SS, Longhi JJ, Goldman CD, McFadden DW (2007) Surgery for obesity: a review of the current state of the art and future directions. J Gastrointest Surg 11:377-397

23. Lujan JA, Frutos MD, Hernandez Q, Liron R, Cuenca JR, Valero $G$ et al (2004) Laparoscopic versus open gastric bypass in the treatment of morbid obesity: a randomized prospective study. Ann Surg 239:433-437

24. Nguyen NT, Goldman C, Rosenquist CJ, Arango A, Cole CJ, Lee SJ et al (2001) Laparoscopic versus open gastric bypass: a randomized study of outcomes, quality of life, and costs. Ann Surg 234:279-289 discussion 289-291

25. DeMaria EJ, Sugerman HJ, Kellum JM, Meador JG, Wolfe LG (2002) Results of 281 consecutive total laparoscopic Roux-en-Y gastric bypasses to treat morbid obesity. Ann Surg 235:640-645 discussion 645-647

26. Wittgrove AC, Clark GW (2000) Laparoscopic gastric bypass, Roux-en-Y- 500 patients: technique and results, with 3-60 month follow-up. Obes Surg 10:233-239

27. Schauer PR, Ikramuddin S, Gourash W, Ramanathan R, Luketich J (2000) Outcomes after laparoscopic Roux-en-Y gastric bypass for morbid obesity. Ann Surg 232:515-529

28. Higa KD, Boone KB, Ho T, Davies OG (2000) Laparoscopic Roux-en-Y gastric bypass for morbid obesity: technique and preliminary results of our first 400 patients. Arch Surg 135:10291033 discussion 1033-1024 